\section{P47 NATIONAL ESTIMATES OF THE ECONOMIC BURDEN OF DIABETES}

KN O'Neill*, SM McHugh, AP Fitzgerald, PM Kearney. School of Public Health, UCC, Cork, Ireland

\subsection{6/jech-2019-SSMabstracts. 198}

Background Valid and reliable estimates for the incremental costs attributable to diabetes at country-level are required to provide more precise estimates of the global cost of diabetes and to encourage and inform national responses to the diabetes epidemic. The most recent estimates for Ireland come from the CODEIRE study which uses data from the years 1999/2000 and employs a sum-all medical approach for a hospital-based sample of people with diabetes. Indirect costs are not estimated We estimate the total costs, direct and indirect, attributable to diabetes in older Irish adults.

Methods Adopting a societal perspective and using a prevalence-based approach, we estimate the direct costs (healthcare utilisation and medications) and indirect costs (participation in the labour force and premature mortality) attributable to diabetes in 2013. A human capital approach was used to estimate indirect costs. Where possible, incremental costing methodology was employed. Data sources include a nationally representative cohort study, a national pharmacy claims database and national vital statistics.

Results The estimated total cost of diabetes was $€ 536,668,060 \quad$ (95\% CI: $360,172,044-751,720,140)$; $€ 238,155,072$ (95\% CI: 192,023,954-278,959,992) in direct costs and $€ 298,512,988$ (95\% CI: 168,148,090-472,760,148) in indirect costs. People with diabetes were $41 \%$ less likely to be employed than those without diabetes $(\mathrm{p}<0.001)$, with the loss in productivity estimated at $€ 190,864,409$ (95\% CI: $104,107,859-294,972,268)$ or $36 \%$ of total costs. Prescription costs were the next largest contributor, calculated at $€ 156,429,993$. The total costs of premature mortality were estimated at $€ 107,648,579 \quad(95 \% \quad$ CI: 64,040,231177,787,880).

Conclusion We establish the societal costs attributable to diabetes in older adults in Ireland and therefore identify costs that can be directly targeted by prevention and treatment strategies. Primary, secondary and tertiary prevention of diabetes can result in significant cost savings, not only in terms of direct healthcare costs but also indirect costs.

\section{P48 WHAT IS THE EVIDENCE BASE FOR INTEGRATING HEALTH AND ENVIRONMENTAL AGENDAS IN THE SCHOOL CONTEXT TO IMPROVE HEALTHY AND ENVIRONMENTALLY AWARE BEHAVIOURS? A SYSTEMATIC SCOPING REVIEW OF GLOBAL EVIDENCE}

${ }^{1} \mathrm{R}$ Proctor* ${ }^{*}{ }^{1} \mathrm{C}$ Guell, ${ }^{2} \mathrm{~K}$ Wyatt, ${ }^{1} \mathrm{AJ}$ Williams. ${ }^{1}$ European Centre for Environment and Human Health, University of Exeter College of Medicine and Health, Truro, UK; ${ }^{2}$ Institute for Health Research, University of Exeter College of Medicine and Health, Exeter, UK

\subsection{6/jech-2019-SSMabstracts. 199}

Background Society is experiencing increasing rates of noncommunicable diseases (NCDs) and environmental degradation related to climate change. Links between behaviours that exacerbate both poor health and carbon emissions such as food and transport choices are related to individual and collective behaviours. As many behaviours are shaped in early life, schools might be important settings in which to address these issues. To explore this further, we examined the global evidence base for integrated strategies for promoting healthy and environmentally aware behaviours within the school context.

Methods A systematic scoping review was undertaken to identify literature on integrated health and environmental agendas in schools over the last 20 years. Search strings were applied across 10 databases covering relevant disciplines. In total, 3051 titles and abstracts were screened and 228 papers underwent a full-text screen by two independent reviewers, verified by a third. Two texts were found via additional citation searches. 87 texts were eligible, 75 journal articles and 12 additional sources including books and grey literature. Texts were included if they explicitly explored combining health and environmental agendas in any school context. No restrictions in terms of study design, aspect of health or population within the school context were applied.

Results Evidence of whether integrating health and environmental agendas in the school context could improve behaviours related to NCDs and climate change has grown since 2004, particularly in the North American, Australasian and Scandinavian context. Out of the 87 included texts, 55 were based on empirical data: primary qualitative (21), quantitative research (6), mixed method (16) or secondary analysis (12), such as systematic reviews (3). A range of disciplinary fields, most notably Education, Public Health and Geography were found to have explored integration. Only 2/55 studies were found to have follow up longitudinal data. Thirteen of the included texts focused on theories or frameworks associated with integration. A further 8 texts presented logic models to explain the components involved in integration. Predominant theories and concepts included: participation pedagogies, action competencies and socio-ecological frameworks.

Conclusion This review found little consistent evidence to support the implementation of integrated strategies; there seems to be a need for developing an interdisciplinary theoretical framework to guide future research, and strengthening the evidence base could translate research and guide future-focused policy and practice. To inform this, a follow up in-depth evidence synthesis will focus on the subset of conceptual texts.

Funding This work was conducted as part of a $\mathrm{PhD}$ project funded by the University of Exeter College of Medicine and Health. Partial funding also provided by the National Institute for Health Research (NIHR) Collaboration for Leadership in Applied Health Research and Care South West Peninsula (NIHR CLAHRC South West Peninsula). The views expressed are those of the author(s) and not necessarily those of the NHS, the NIHR or the Department of Health and Social Care.

\section{P49 BIRTHWEIGHT, LIFETIME OBESITY AND PHYSICAL FUNCTIONING IN MID-ADULTHOOD: A NATIONWIDE BIRTH COHORT STUDY}

${ }^{1}$ NT Rogers*, ${ }^{2} \mathrm{C}$ Power, ${ }^{1}$ SP Pereira. ${ }^{1}$ UCL Research Department of Epidemiology and Public Health, University College London, London, UK; ${ }^{2}$ Population, Policy and Practice, UCL Great Ormond Street Institute of Child Health, London, UK

\subsection{6/jech-2019-SSMabstracts.200}

Background Physical functioning from mid-adulthood is important for maintaining independence and is linked to better health outcomes. Evidence is scant on long-term implications of childhood obesity and body mass index (BMI) gains over the life-course for poor physical functioning $(\mathrm{PF})$ in midlife. 
Studies that link obesity to poor PF have typically been crosssectional, limited to two time-points or relied on retrospectively recalled height and weight. We aimed to establish associations between PF at age 50y and i) birthweight and BMI across the life-course; ii) BMI gains at specific life-stages and iii) age of obesity onset.

Methods The 1958 birth cohort includes all born in one week, in March 1958, across Britain. BMI (kg/m2) was calculated ( $\mathrm{N}=4,173$ (males); 4,501 (females)) using height and weight measured at school $(7,11,16 y)$, in participant's homes $(33,45 \mathrm{y})$ or self-reported $(23,50 \mathrm{y})$. PF at $50 \mathrm{y}$ was assessed via postal questionnaire using the validated $\mathrm{PF}$ subscale of the Short-form 36 (SF-36) survey. The lowest sex-specific 10th centile were defined as poor PF. Missing data was imputed via multiple imputation. Associations were examined using logistic regression, adjusting for social class, education and health behaviours.

Results Birthweight was not associated with PF. At each adult age, odds of poor PF were highest for obese (vs normal) e.g. for $23 \mathrm{y}$ obesity the $\mathrm{OR}_{\text {adjusted }}$ for poor $\mathrm{PF}$ was 2.28 $(1.34,3.91)$ and $2.67(1.72,4.14)$ in males and females respectively. BMI gains from adolescence were related to poor PF, e.g. for females, $\mathrm{OR}_{\text {adjusted }}$ per $\mathrm{SD}$ in $\mathrm{BMI}$ gain between 16$23 y$ was $1.28(1.13,1.46)$; for BMI gains $45-50 y$ it was 1.36 $(1.11,1.65)$. Mean BMI at 50y increased with earlier onset of obesity; e.g. in males, from $31.4 \mathrm{~kg} / \mathrm{m}^{2}$ for mid-adult onset to $35.1 \mathrm{~kg} / \mathrm{m}^{2}$ for child onset. Longer duration of obesity was associated with poor PF (p-trend<0.001), e.g. in males, for childhood obesity onset (vs. never obese) $\mathrm{OR}_{\text {adjusted }}$ was 2.32 $(1.26,4.29)$; for mid-adulthood obesity onset it was $1.50(1.16$, 1.96); associations were abolished with further adjustment for $50 \mathrm{y}$ BMI.

Conclusion Study strengths include the large nationwide cohort followed from birth and prospective measures of BMI and PF, albeit BMI at some ages was self-reported. Obesity, BMI gains and earlier obesity onset were associated with poor $\mathrm{PF}$ in mid-adulthood. Findings relating to duration of obesity are important given the increasing prevalence of childhood obesity, which tends to track into adulthood. Our study highlights the importance of preventing and delaying obesity onset to mitigate the risk of poor PF in mid-adulthood.

\section{P50 PREDICTIVE FACTORS FOR INDETERMINATE RESULTS IN INTERFERON- $\gamma$ RELEASE ASSAYS - A PROBLEM TO CONSIDER}

\begin{abstract}
1,2,3 JA Santos* ${ }^{*}, 5 \mathrm{R}$ Duarte, ${ }^{1,3} \mathrm{C}$ Nunes. ${ }^{1}$ Escola Nacional de Saúde Pública, Universidade NOVA de Lisboa; ${ }^{2}$ Instituto Nacional de Saude Dr. Ricardo Jorge, Lisboa, Portugal; ${ }^{3}$ Centro de Investigação em Saúde Pública, Lisboa, Portugal; ${ }^{4}$ Faculdade de Medicina da Universidade do Porto, Porto, Portugal; ${ }^{5}$ Centro Diagnóstico Pneumológico de Vila Nova de Gaia, Porto, Portugal
\end{abstract}

\subsection{6/jech-2019-SSMabstracts.201}

Background Interferon- $\boldsymbol{\gamma}$ release assays (IGRA) are a crucial diagnostic tool for the detection of a Mycobacterium tuberculosis infection in order to control and eliminate the tuberculosis (TB) epidemic. Indeterminate results can occur and represent a considerable problem for clinical management, since they imply the lack of clear information about the patient's TB infection status. The aim of the study was to identify risk factors that could be associated with indeterminate IGRA results.
Methods Retrospective cohort study carried out using data from the Portuguese National Tuberculosis Surveillance system, from 2008 to 2015 . Were included in the study 1230 patients with active TB and an IGRA result. The IGRA test used in the patients enrolled in the study was the QuantiFERON-TB Gold In-Tube (Qiagen). The association between indeterminate IGRA results and sociodemographic factors, comorbidities and the site of disease were evaluated through bivariate and multivariate logistic regression analysis.

Results Of the 1230 patients reported with active TB in the SVIG-TB database (2008-2015) that underwent an IGRA test, 857 patients $(69.7 \%)$ had a positive test result, $212(17.2 \%)$ had a negative result and 161 (13.1\%) had an indeterminate result. Majority of the patients with indeterminate results were male $(67.7 \%)$ and more than half had more than 50 years (57.1\%). The proportion of indeterminate results increased as the age increased, with patients over 80 years old presenting the highest proportion of indeterminate results. Age $\geq 65$ years (OR 2.51, p<0.001), alcohol abuse (OR 3.04, p=0.001) and pulmonary TB (OR 3.07, p<0.001) were predictive factors for indeterminate IGRA results.

Conclusion Age $\geq 65$ years, alcohol abuse and pulmonary TB were identified as factors for the occurrence of indeterminate IGRA results. The first two factors can be identified prior to the test and thus help to quickly identify the probable cause of an indeterminate outcome and lead to the use of other clinical and diagnostic means to detect a possible infection.

\section{P51 SENSITIVITY AND AGREEMENT OF AN INTERFERON- GAMMA RELEASE ASSAY AND TUBERCULIN SKIN TEST IN PATIENTS WITH PULMONARY AND EXTRAPULMONARY TUBERCULOSIS}

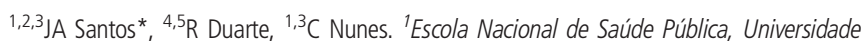
NOVA de Lisboa, Portugal; ${ }^{2}$ Instituto Nacional de Saude Dr. Ricardo Jorge, Lisboa, Portugal; ${ }^{3}$ Centro de Investigação em Saude Publica, Lisboa, Portugal; ${ }^{4}$ Faculdade de Medicina da Universidade do Porto, Porto, Portugal; ${ }^{5}$ Centro Diagnóstico Pneumológico de Vila Nova de Gaia, Porto, Portugal
\end{abstract}

\subsection{6/jech-2019-SSMabstracts.202}

Background Tuberculosis (TB) is still a serious global public health concern, being essential a rapid and accurate diagnosis of infected individuals. The aim of this study was to estimate the sensitivity of the interferon- $\gamma$ release assays (IGRA) and tuberculin skin test (TST) in patients diagnosed with active TB and the agreement between the tests.

Methods Retrospective cohort study carried out using data from the Portuguese National Tuberculosis Surveillance system, from 2008 to 2015. The study included all TB cases with an IGRA and TST result $(n=727)$. The IGRA test used in the patients enrolled in the study was the QuantiFERON-TB Gold In-Tube (Qiagen). Sensitivity was calculated with 95\% confidence interval $(95 \% \mathrm{CI})$ for each test separately and in combination (IGRA and TST-5 mm or IGRA and TST-10 mm) and outcomes were compared using McNemar's test. Kappa coefficient $(\mathrm{k})$ was used to evaluate the agreement between IGRA and TST test results.

Results The mean age of the patients was 47.9 years ( \pm standard deviation 20.0 years), ranging from less than a 1 year to 91 years, with the age group 16-64 years representing the majority of cases. IGRA, TST-5 mm and TST-10 mm were positive in $82.4 \%, 84.5 \%$ and $78.4 \%$ of the $\mathrm{TB}$ patients. These results imply that $128(17.6 \%), 112(15.4 \%)$ and 157 